\title{
Nonlinear Control of Bioprocess Using Feedback Linearization, Backstepping, and Luenberger Observers
}

\author{
Muhammad Rizwan S. Khan, Robert N. K. Loh \\ Department of Electrical and Computer Engineering, Oakland University, Rochester, Michigan, USA \\ Email: mskhan@oakland.edu, loh@oakland.edu
}

Received 12 July 2014; revised 15 August 2014; accepted 29 August 2014

Copyright (C) 2014 by authors and Scientific Research Publishing Inc.

This work is licensed under the Creative Commons Attribution International License (CC BY).

http://creativecommons.org/licenses/by/4.0/

c) (i) Open Access

\begin{abstract}
This paper addresses the analysis, design, and application of observer-based nonlinear controls by combining feedback linearization (FBL) and backstepping (BS) techniques with Luenberger observers. Complete development of observer-based controls is presented for a bioprocess. Controllers using input-output feedback linearization and backstepping techniques are designed first, assuming that all states are available for feedback. Next, the construction of observer in the transformed domain is presented based on input-output feedback linearization. This approach is then extended to observer design based on backstepping approach using the error equation resulted from the backstepping design procedure. Simulation results demonstrating the effectiveness of the techniques developed are presented and compared.
\end{abstract}

\section{Keywords}

Bioprocess, Feedback Linearization, Backstepping, Luenberger Observers, Observer-Based Control

\section{Introduction}

In process control, a major difficulty is to provide direct real-time measurements of the state variables required to implement advanced monitoring and control methods on bioreactors [1]-[5]. Dissolved oxygen concentration in bioreactors, temperature in non-isothermal reactors and gaseous flow rates, are available for on-line measurement while the values of concentration of products, reactants and/or biomass are often available only via on-line analysis [2]-[4], which means that these variables are not available for real-time feedback control. An alternative is to use state observers which, in conjunction with the process model and available measurements, 
can generate accurate estimates of the unmeasured and/or inaccessible states effectively. Exponential and asymptotic observers and their variants to estimate unmeasured states in bioprocess systems have appeared in [1]-[5]. In [6], Dochain and Perrier applied backstepping [7]-[9], techniques to the nonlinear control of microbial growth problem in a CSTR (continuously stirred tank reactor) and two controllers were proposed. The first one was a non-adaptive version, while the second one was an adaptive version in which the maximum specific growth rate was estimated on-line. However, backstepping-based observer design was not considered in [6].

In this paper, a complete development of observer-based control is presented that includes feedback linearization [7] [8] [10] [11], backstepping [7]-[9], Luenberger observer [12] with feedback linearization, and Luenberger observer with backstepping.

The paper is organized as follows. Section 2 presents the bioprocess model for control design. Theoretical foundation of input-output feedback linearization (FBL) and controller design are outlined in Section 3 with simulation results. Section 4 addresses the formulation and application of backstepping (BS) control with simulation results. In Section 5, simulation results are compared for both approaches, i.e., FBL and BS. Section 6 addresses the design of Luenberger observers for FBL and BS controls with simulations. The conclusions are presented in Section 7.

\section{Bioprocess Model}

The model dynamics in a CSTR (continuous stirred tank reactor) with a simple microbial growth reaction, with one substrate $S$ and biomass $X$, are given by the following equations [1]:

$$
\begin{gathered}
\frac{\mathrm{d} X}{\mathrm{~d} t}=\mu X-D X, \\
\frac{\mathrm{d} S}{\mathrm{~d} t}=-k_{1} \mu X+D S_{\text {in }}-D S,
\end{gathered}
$$

where $k_{1}, \mu, D, S, S_{\text {in }}$, represent the yield coefficient, specific growth rate $\left(\mathrm{h}^{-1}\right)$, dilution rate $\left(\mathrm{h}^{-1}\right)$, and substrate concentration (grams/lit) in the influent and reactor, respectively.

The biomass concentration $X(t)$ (grams/lit) is the variable which is to be controlled. Defining the parameter $\theta_{1}$ as $\theta_{1}=D / k_{o}$ and expressing specific growth rate $\mu$ as $\mu=k_{o} r(s)$, the dynamical Equations (1) and (2) above can be written as [6]

$$
\left\{\begin{array}{l}
\dot{x}_{1}=\theta_{1}^{-1} r\left(x_{2}\right) x_{1}-x_{1}, \\
\dot{x}_{2}=-k_{1} \theta_{1}^{-1} r\left(x_{2}\right) x_{1}-x_{2}+u, \\
y=x_{1},
\end{array}\right.
$$

where it is assumed that the biomass concentration $x_{1}(t) \triangleq X(t)$ can be measured with a sensor, i.e., the output is given by $y=x_{1}$, while $x_{2}(t) \triangleq S(t)$ denotes the substrate concentration and $u(t) \triangleq S_{\text {in }}$ is the control input. The bioprocess model given by (3) can be written compactly in an alternate state-space form as:

$$
\left\{\begin{array}{l}
{\left[\begin{array}{l}
\dot{x}_{1} \\
\dot{x}_{2}
\end{array}\right]=\left[\begin{array}{l}
\frac{C_{1} x_{1} x_{2}}{K_{s}+x_{2}}-x_{1} \\
\frac{C_{2} x_{1} x_{2}}{K_{s}+x_{2}}-x_{2}
\end{array}\right]+\left[\begin{array}{l}
0 \\
1
\end{array}\right] u \triangleq \boldsymbol{f}(\boldsymbol{x})+\boldsymbol{g}(\boldsymbol{x}) u,} \\
y=x_{1}=\left[\begin{array}{ll}
1 & 0
\end{array}\right] \boldsymbol{x} \triangleq \boldsymbol{C} \boldsymbol{x},
\end{array}\right.
$$

where $C_{1} \triangleq \frac{\mu_{\max }}{D}$ and $C_{2} \triangleq-k_{1} \frac{\mu_{\max }}{D}$. Note that in (4), $r\left(x_{2}\right)$ has been written using "Monod form" for reaction kinetics, which can expressed as

$$
r\left(x_{2}\right)=\frac{\mu_{\max }}{k_{o}} \frac{x_{2}}{K_{S}+x_{2}}
$$




$$
\Rightarrow \frac{\partial r}{\partial x_{2}}=\frac{\mu_{\max }}{k_{o}} \frac{K_{S}}{\left(K_{S}+x_{2}\right)^{2}} .
$$

We will use (3) for back stepping control and observer design and (4) will be utilized for developing the control law and observer design using the feedback linearization approach. Typical values of the model parameters needed for the simulation studies are given in Table 1 [6].

\section{Feedback Linearization (FBL) Control Design}

The main intent of this section is to investigate control design using the input-output feedback linearization (FBL) technique. Consider a general nonlinear control-affine SISO system described by [7] [8] [10] [11],

$$
\begin{gathered}
\dot{\boldsymbol{x}}=\boldsymbol{f}(\boldsymbol{x})+\boldsymbol{g}(\boldsymbol{x}) u, \quad \boldsymbol{f}, \boldsymbol{g}: D \subset \mathbb{R}^{n} \rightarrow \mathbb{R}^{n}, \\
y=h(\boldsymbol{x}), \quad h: D \subset \mathbb{R}^{n} \rightarrow \mathbb{R},
\end{gathered}
$$

where $\boldsymbol{x} \in \mathbb{R}^{n}$ is the state vector, $u, y \in \mathbb{R}$ are the control and output signals, respectively; $h$ is a smooth function, and $\boldsymbol{f}, \boldsymbol{g}$ are smooth vector fields on $D$, where $D$ is an open set. Given the nonlinear system (7) and the measurement (8), our goal is to find a diffeomorphism or nonlinear transformation of the form $\mathbf{z}=\boldsymbol{T}_{f b l}(\boldsymbol{x})$, $\mathbf{z} \in \mathbb{R}^{n}$ with $\boldsymbol{T}_{f b l}(\mathbf{0})=\mathbf{0}$ that transforms the nonlinear system in the $x$-coordinates to a linear system in the $z$-coordinates. Differentiating the output $y(t)$ with respect to $t$ yields

$$
\dot{y}=L_{f} h(x)+L_{g} h(x) u,
$$

where $L_{f} h(\boldsymbol{x})=\frac{\partial h}{\partial \boldsymbol{x}} \boldsymbol{f}$ and $L_{\boldsymbol{g}} h(\boldsymbol{x})=\frac{\partial \boldsymbol{h}}{\partial \boldsymbol{x}} \boldsymbol{g}$ denote the Lie derivatives of $h(\boldsymbol{x})$ with respect to $\boldsymbol{f}(\boldsymbol{x})$ and $\boldsymbol{g}(\boldsymbol{x})$, respectively. If $L_{\boldsymbol{g}} h(\boldsymbol{x})=0$, then $\dot{y}(t)$ is independent of $u(t)$. Continuing successive differentiation $\rho$ times until $u(t)$ appears explicitly, we obtain

$$
y^{(\rho)}=L_{f}^{\rho} h(x)+L_{g} L_{f}^{\rho-1} h(x) u \triangleq \alpha(x)+\beta(x) u,
$$

where $\alpha(\boldsymbol{x}) \triangleq L_{f}^{\rho} h(\boldsymbol{x})$ is a nonlinearity cancellation factor and $\beta(\boldsymbol{x}) \triangleq L_{g} L_{f}^{\rho-1} h(\boldsymbol{x})$ is a scalar function. The smallest integer $\rho$ for which $u(t)$ appears is referred to as the relative degree, i.e., when $L_{g} L_{f}^{\rho-1} h(x) \neq 0$. The nonlinear system (7) - (8) is said to have a well-defined relative degree $\rho$ in a region $D_{0} \subset D$ if

$$
\left\{\begin{array}{l}
L_{g} L_{f}^{k} h(x)=0 \quad \forall k, 0 \leq k<\rho-1 ; \\
L_{g} L_{f}^{\rho-1} h(x) \neq 0,
\end{array}\right.
$$

for all $\boldsymbol{x} \in D_{0}$. Note that $\rho \leq n$. From (10), define

$$
v \triangleq y^{(\rho)}=\alpha(\boldsymbol{x})+\beta(\boldsymbol{x}) u,
$$

where $v(t)$ is a one-dimensional transformed input created by the feedback linearization process. Equation (12) yields the linearizing feedback control law [7] [8] [10] [11]:

$$
u=\beta^{-1}(x)[-\alpha(x)+v],
$$

Table 1. Parameter data.

\begin{tabular}{ccc}
\hline Symbol & Parameter & Numerical Value \\
\hline$k_{1}$ & Yield coefficient & 2 \\
$\mu_{\max }$ & Specific growth rate & $0.33 \mathrm{~h}^{-1}$ \\
$D$ & Dilution rate & $0.05 \mathrm{~h}^{-1}$ \\
$K_{s}$ & Saturation constant & 5 grams/lit \\
\hline
\end{tabular}


provided $\beta(\boldsymbol{x})$ is invertible. If $\rho=n$, then

$$
\boldsymbol{z}=\boldsymbol{T}_{f b l}(\boldsymbol{x})=\left[\begin{array}{c}
h(\boldsymbol{x}) \\
L_{f} h(\boldsymbol{x}) \\
\vdots \\
L_{f}^{n-1} h(\boldsymbol{x})
\end{array}\right] .
$$

If $\rho<n$, the diffeomorphism $\boldsymbol{T}_{f b l}(\boldsymbol{x})$ comprises of both external and internal dynamics, i.e., $\boldsymbol{T}_{f b l}(\boldsymbol{x})=[\boldsymbol{\xi} \mid \boldsymbol{\eta}]^{\mathrm{T}}$, where $\boldsymbol{\xi} \in \mathbb{R}^{\rho}$ represents the external dynamics state vector and $\boldsymbol{\eta} \in \mathbb{R}^{n-\rho}$ the internal dynamics state vector, respectively; furthermore, the differential equation for $\boldsymbol{\xi}$ is linear, while that for $\boldsymbol{\eta}$ is typically nonlinear. For the bioprocess model given by (4), $\rho=n=2$, so the system is fully linearizable. We obtain, from (4), (12) and (14),

$$
\begin{gathered}
\alpha(\boldsymbol{x})=\frac{\left(C_{1} x_{2}-K_{s}-x_{2}\right)\left(C_{1} x_{1} x_{2}-K_{s} x_{1}-x_{1} x_{2}\right)}{\left(K_{s}+x_{2}\right)^{2}}+\frac{C_{1} C_{2} K_{S} x_{1}^{2} x_{2}}{\left(K_{s}+x_{2}\right)^{3}}-\frac{C_{1} K_{S} x_{1} x_{2}}{\left(K_{s}+x_{2}\right)^{2}}, \\
\beta(\boldsymbol{x})=\frac{C_{1} K_{s} x_{1}}{\left(K_{s}+x_{2}\right)^{2}}, \\
\mathbf{z}=\boldsymbol{T}_{f b l}(\boldsymbol{x})=\left[\begin{array}{c}
y \\
\dot{y}
\end{array}\right]=\left[\begin{array}{c}
x_{1} \\
\frac{C_{1} x_{1} x_{2}}{K_{s}+x_{2}}-x_{1}
\end{array}\right],
\end{gathered}
$$

where $\boldsymbol{T}_{f b l}(\boldsymbol{x})$ is a local diffeomorphism for the system. Using (17) with (12) for $\rho=2$, the original nonlinear system described by (3) and (4) is transformed into a linear system of the form

$$
\left\{\begin{array}{l}
\dot{\mathbf{z}}=\boldsymbol{A}_{c} \mathbf{z}+\boldsymbol{B}_{c} v, \quad \mathbf{z}(0)=\mathbf{z}_{o}, \\
y=\boldsymbol{C}_{c} \mathbf{z}
\end{array}\right.
$$

where

$$
\left\{\begin{array}{l}
\boldsymbol{A}_{c}=\left[\begin{array}{ll}
0 & 1 \\
0 & 0
\end{array}\right], \quad \boldsymbol{B}_{c}=\left[\begin{array}{l}
0 \\
1
\end{array}\right], \quad \boldsymbol{C}_{c}=\left[\begin{array}{ll}
1 & 0
\end{array}\right], \\
y=\boldsymbol{C}_{c} \mathbf{z},
\end{array}\right.
$$

and $\left[\boldsymbol{A}_{c}, \boldsymbol{B}_{c}\right]$ and $\left[\boldsymbol{A}_{c}, \boldsymbol{C}_{c}\right]$ are, respectively, controllable and observable pairs. A suitable tracking control law for the transformed input $v(t)$ in the linear system (18) for $\rho=2$ can be formulated as, with (17),

$$
v=-\boldsymbol{K}_{f b l} \mathbf{Z}+\boldsymbol{K}_{f b l} \boldsymbol{Y}_{r}+\ddot{y}_{r}=-\boldsymbol{K}_{f b l} \boldsymbol{T}_{f b l}(\boldsymbol{x})+\boldsymbol{K}_{f b l} \boldsymbol{Y}_{r}+\ddot{y}_{r},
$$

where $\mathbf{z}=\boldsymbol{T}_{f b l}(\boldsymbol{x}), y_{r}(t)$ is a bounded reference with bounded derivatives $\dot{y}_{r}(t)$ and $\ddot{y}_{r}(t), \boldsymbol{Y}_{r}=\left[\begin{array}{ll}y_{r} & \dot{y}_{r}\end{array}\right]^{\mathrm{T}}$, and the constant feedback gain matrix $\boldsymbol{K}_{f b l}=\left[\begin{array}{ll}K_{1 f b l} & K_{2 f b l}\end{array}\right]$ is determined such that $\left(\boldsymbol{A}_{c}-\boldsymbol{B}_{c} \boldsymbol{K}_{f b l}\right)$ is Hurwitz. Furthermore, the gain matrix $\boldsymbol{K}_{f b l}$ can be determined by various design methods, such as pole placement (PP) and linear quadratic regulator (LQR). We shall focus on the PP design in this paper. Substituting (20) into (18) yields the closed-loop system

$$
\left\{\begin{array}{l}
\dot{\boldsymbol{z}}=\left(\boldsymbol{A}_{c}-\boldsymbol{B}_{c} \boldsymbol{K}_{f b l}\right) \mathbf{z}+\boldsymbol{B}_{c}\left(\boldsymbol{K}_{f b l} \boldsymbol{Y}_{r}+\ddot{y}_{r}\right), \quad \mathbf{z}(0)=\mathbf{z}_{o}, \\
y=\boldsymbol{C}_{c} \mathbf{z} .
\end{array}\right.
$$

The linearizing feedback control law in the $x$-domain can be written by setting $u=u_{f b l}$ in (13) as

$$
u_{f b l}=\beta^{-1}(\boldsymbol{x})[-\alpha(\boldsymbol{x})+v]=\beta^{-1}(\boldsymbol{x})\left[-\alpha(\boldsymbol{x})-\boldsymbol{K}_{f b l} \boldsymbol{T}_{f b l}(\boldsymbol{x})+\boldsymbol{K}_{f b l} \boldsymbol{Y}_{r}+\ddot{y}_{r}\right],
$$

which yields the closed-loop system 


$$
\left\{\begin{array}{l}
\dot{\boldsymbol{x}}=\boldsymbol{f}(\boldsymbol{x})+\boldsymbol{g}(\boldsymbol{x}) u_{f b l}, \quad \boldsymbol{x}(0)=x_{o}, \\
y=x_{1} .
\end{array}\right.
$$

The design of a PP control law (20) for the $2^{\text {nd }}$-order system (18) is achieved by choosing a damping ratio $\xi=1.0$ that prohibits overshoot, and an undamped natural frequency $\omega_{n}=8.2(\mathrm{rad} / \mathrm{sec})$. The resulting closedloop poles are given by $\boldsymbol{p}=\left[-\xi \omega_{n} \pm j \omega_{d}\right]=\left[\begin{array}{ll}-8.2 & -8.2\end{array}\right]$ where $\omega_{d}=\omega_{n} \sqrt{1-\xi^{2}}=0$ and the resulting gain $\boldsymbol{K}_{f b l}=\left[\begin{array}{ll}67.24 & 16.4\end{array}\right]$ is computed with Matlab's ACKER command. Simulation studies for the closed-loop system with FBL control were conducted using (23). The controller performance was evaluated for a square-wave set-point reference $y_{r}(t)$ that alternates every 20 hours between 3 grams/lit and 4 grams/lit as shown in Figure 1 (dotted line). The initial conditions were chosen as $X(0)=x_{1}(0)=2$ grams/lit and $S(0)=x_{2}(0)=0.9$ [6]. The simulation results are depicted in Figure 1 which shows that the responses are satisfactory.

\section{Backstepping (BS) Control Design}

We shall address the design of back stepping (BS) [7]-[9] control in this section, where the parameter $\theta_{1}$ is assumed known. The objective here is to design a BS control law $u_{b s}$ such that the output $y=x_{1}$ tracks the reference $y_{r}$. We will also compare the performance of the closed-loop bioprocess under FBL control $u_{f b l}$ given by (22) and the BS control $u_{b s}$ to be developed below. The formulation presented here considers a general bounded differentiable reference signal $y_{r}(t)$ instead of the constant set-point regulation in [6]. Consider the nonlinear system in the form of (3) reproduced below for ease of reference:

$$
\left\{\begin{array}{l}
\dot{x}_{1}=\theta_{1}^{-1} r\left(x_{2}\right) x_{1}-x_{1}, \\
\dot{x}_{2}=-k_{1} \theta_{1}^{-1} r\left(x_{2}\right) x_{1}-x_{2}+u, \\
y=x_{1},
\end{array}\right.
$$

where $\theta_{1}$ is a known constant parameter. We treat $r\left(x_{2}\right) x_{1}$ as the virtual control of the first subsystem $\dot{x}_{1}=\theta_{1}^{-1} r\left(x_{2}\right) x_{1}-x_{1}$ in (3) and let $\alpha_{1}$ be the stabilizing function such that $y=x_{1}$ racks $y_{r}$. Define the tracking errors as

$$
\begin{aligned}
& q_{1}=y-y_{r}=x_{1}-y_{r}, \\
& q_{2}=\theta_{1}^{-1} r\left(x_{2}\right) x_{1}-\alpha_{1},
\end{aligned}
$$

(a)

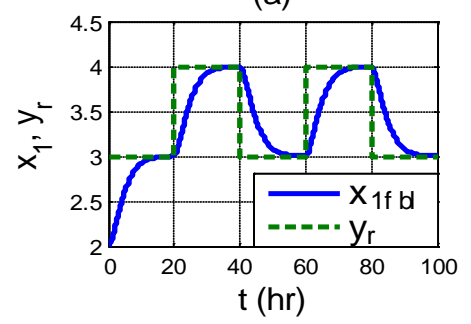

(c)

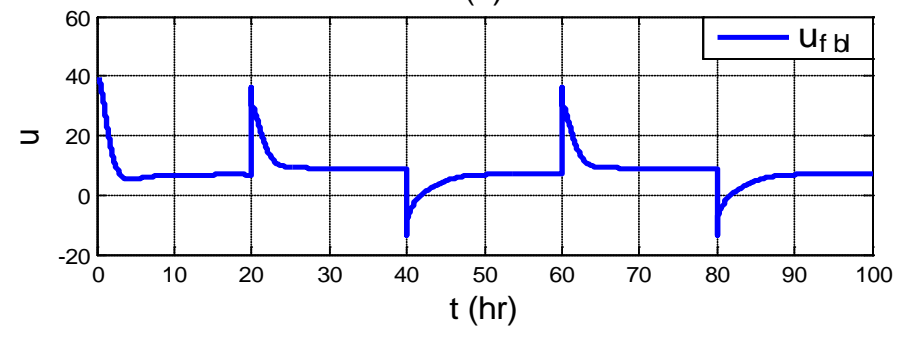

Figure 1. Responses of closed-loop bioprocess (23) under FBL control $u_{f b l}(t)$ with PP design.

(b)

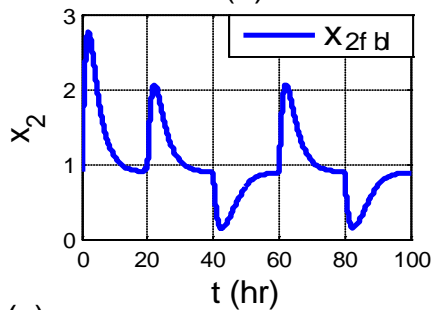


where $q_{2}$ is the error between $\theta_{1}^{-1} r\left(x_{2}\right) x_{1}$ and $\alpha_{1}$. Taking the derivative of $q_{1}$ yields, with (25),

$$
\dot{q}_{1}=\dot{x}_{1}-\dot{y}_{r}=\theta_{1}^{-1} r\left(x_{2}\right) x_{1}-x_{1}-\dot{y}_{r}=q_{2}+\alpha_{1}-x_{1}-\dot{y}_{r} .
$$

Consider the Lyapunov function candidate

$$
V_{1}=\frac{1}{2} q_{1}^{2}
$$

which yields the derivative, with (26),

$$
\dot{V}_{1}=q_{1} q_{2}+q_{1}\left(\alpha_{1}-x_{1}-\dot{y}_{r}\right) .
$$

Choosing the stabilizing function $\alpha_{1}$ to make $\alpha_{1}-x_{1}-\dot{y}_{r}=-c_{1} q_{1}$ in (28) yields

$$
\alpha_{1}=-c_{1} q_{1}+x_{1}+\dot{y}_{r}, \quad c_{1}>0 .
$$

Substituting (29) into (26) and (28) yields, respectively,

$$
\begin{gathered}
\dot{q}_{1}=-c_{1} q_{1}+q_{2}, \\
\dot{V}_{1}=q_{1} q_{2}-c_{1} q_{1}^{2}, \quad \forall c_{1}>0 .
\end{gathered}
$$

From (31), if $q_{2}=0$, then $\dot{V}_{1}=-c_{1} q_{1}^{2}<0 \quad \forall q_{1} \neq 0$ and the origin $q_{1}=0$ is globally asymptotically stable, whereby achieving global tracking with $y=x_{1} \rightarrow y_{r}$. The term $q_{1} q_{2}$ will be addressed in the next step.

The next step is to develop a BS control law for $u$. The derivative of $q_{2}=\theta_{1}^{-1} r\left(x_{2}\right) x_{1}-\alpha_{1}$ given by (25) satisfies, with (3) and (29),

where

$$
\begin{aligned}
\dot{q}_{2} & =\theta_{1}^{-1} r\left(x_{2}\right) \dot{x}_{1}+\theta_{1}^{-1} x_{1} \frac{\partial r}{\partial x_{2}} \dot{x}_{2}-\dot{\alpha}_{1}, \\
& =\phi(x)-\dot{\alpha}_{1}+\theta_{1}^{-1} x_{1} \frac{\partial r}{\partial x_{2}} u,
\end{aligned}
$$

$$
\phi(\boldsymbol{x}) \triangleq \theta_{1}^{-1} r\left(\theta_{1}^{-1} r-1\right) x_{1}+\theta_{1}^{-1} x_{1} \frac{\partial r}{\partial x_{2}}\left(-k_{1} \theta_{1}^{-1} r x_{1}-x_{2}\right)
$$

and $\frac{\partial r}{\partial x_{2}}$ is given by (6).

To stabilize the $\left(q_{1}, q_{2}\right)$-system the Lyapunov function candidate as

$$
V_{2}=V_{1}+\frac{1}{2} q_{2}^{2}
$$

The derivative of $V_{2}$ is given by, with (31) and (33),

$$
\dot{V}_{2}=-c_{1} q_{1}^{2}+q_{2}\left[q_{1}+\phi(x)-\dot{\alpha}_{1}+\theta_{1}^{-1} x_{1} \frac{\partial r}{\partial x_{2}} u\right] .
$$

Defining $u_{b s} \triangleq u$ to be the BS control, and choosing $u_{b s}$ to make the term $[\cdot]=-c_{2} q_{2}$ in (36) yields

$$
u_{b s}=\left(\theta_{1}^{-1} x_{1} \frac{\partial r}{\partial x_{2}}\right)^{-1}\left[-c_{2} q_{2}-q_{1}-\phi(x)+\dot{\alpha}_{1}\right] .
$$

Substituting (37) into (33) and (36), we obtain,

$$
\dot{q}_{2}=-q_{1}-c_{2} q_{2},
$$




$$
\dot{V}_{2}=-c_{1} q_{1}^{2}-c_{2} q_{2}^{2}, \quad c_{1}>0, \quad c_{2}>0 .
$$

Since $\dot{V}_{2}<0$ for all $c_{1}>0, c_{2}>0$, it follows that $\left(q_{1}, q_{2}\right)=(0,0)$ is globally asymptotically stable. Additionally, the stability result can also be established by combing the error equations from (30) and (38) as

$$
\left[\begin{array}{l}
\dot{q}_{1} \\
\dot{q}_{2}
\end{array}\right]=\left[\begin{array}{cc}
-c_{1} & 1 \\
-1 & -c_{2}
\end{array}\right]\left[\begin{array}{l}
q_{1} \\
q_{2}
\end{array}\right] \Rightarrow \dot{\boldsymbol{q}}=\boldsymbol{A}_{q} \boldsymbol{q} .
$$

Since $\boldsymbol{A}_{q}$ is a skew-symmetric Hurwitz matrix for all $c_{1}>0$ and $c_{2}>0$, it follows that the equilibrium $\left(q_{1}, q_{2}\right)=(0,0)$ is globally asymptotically stable. Moreover, $\left[\boldsymbol{A}_{q}, \boldsymbol{C}_{q}\right]$ is an observable pair, where $\boldsymbol{C}_{q}=\left[\begin{array}{ll}1 & 0\end{array}\right]$ (see (53)). Since (40) is in the form of a standard linear time-invariant (LTI) system, a Luenberger observer [12] for state estimation can be constructed for the system, and will be investigated in Section 6 .

Meanwhile, the closed-loop bioprocess under BS control is given by,

$$
\left\{\begin{array}{l}
\dot{\boldsymbol{x}}=\boldsymbol{f}(\boldsymbol{x})+\boldsymbol{g}(\boldsymbol{x}) u_{b s}, \quad \boldsymbol{x}(0)=\boldsymbol{x}_{o}, \\
y=h(\boldsymbol{x})=x_{1},
\end{array}\right.
$$

where $u_{b s}$ is given by (37).

Simulation studies were conducted using (41) with the backstepping gains $c_{1}=c_{2}=8$. The reference signal $y_{r}(t)$ and the initial condition $\boldsymbol{x}(0)=\boldsymbol{x}_{o}$ were same as those used for the FBL control in Section 3. The simulation is depicted in Figure 2 which shows that the responses were satisfactory.

\section{Comparison of FBL and BS Designs}

The simulation results for the FBL versus BS designs using the gains reported in Sections 3 and 4 are shown in Figure 3 and Figure 4 for comparison purposes.

It can be seen that both $x_{1 f b l}(t) \rightarrow y_{r}(t)$ and $x_{1 b s}(t) \rightarrow y_{r}(t)$ asymptotically with no overshoot. It can also be seen that the magnitudes of $u_{f b l}(t)$ are slightly larger than those of $u_{b s}(t)$. However, the reverse can also be obtained by tuning $\boldsymbol{K}_{f b l}$ and $\left\{c_{1}, c_{2}\right\}$.

\section{Observer-Based FBL and BS Controls}

As mentioned before that not all state variables are measured in the bioreactor systems; therefore, suitable observers are needed for realizing the full-state feedback control designs proposed in Sections 3 and 4. We shall

(a)

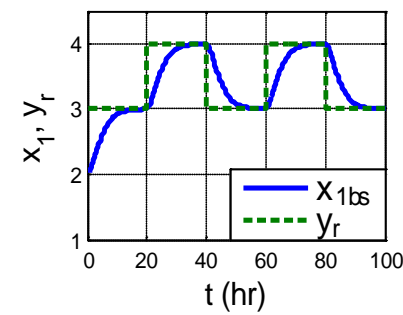

(c)

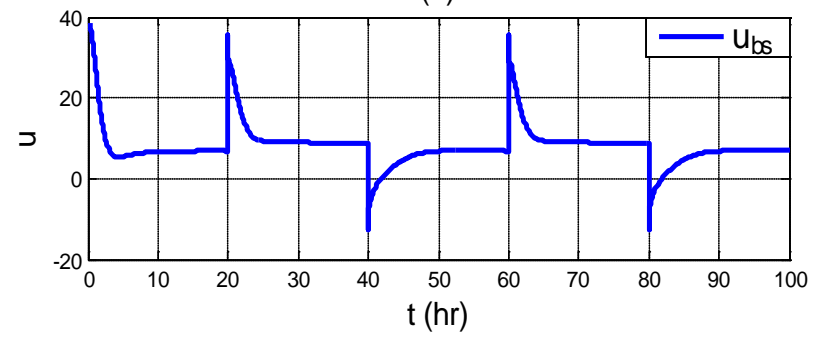

Figure 2. Responses of closed-loop bioprocess (41) under BS control $u_{b s}(t)$ for $c_{1}=c_{2}=8$.

(b)

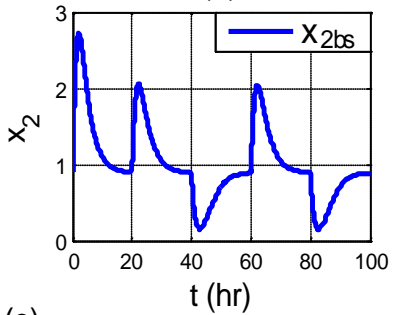$$
\text { 西 }
$$ 
(a)

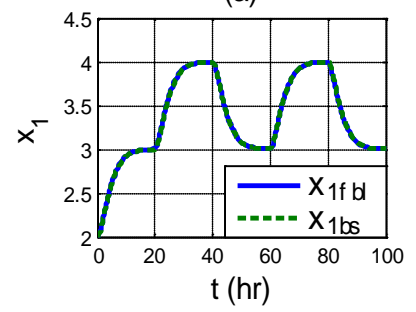

(c)
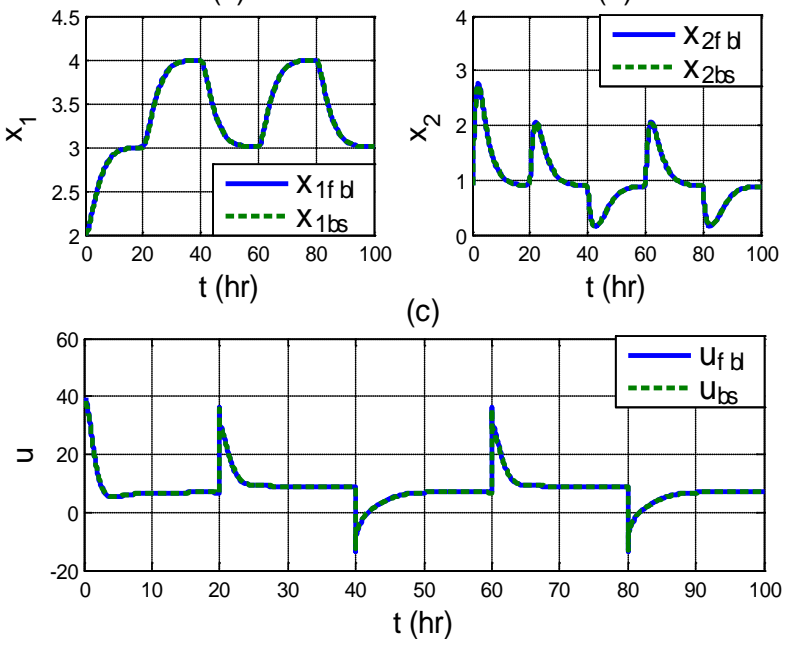

Figure 3. Comparing FBL responses in Figure 1 and BS responses in Figure 2.

(a)

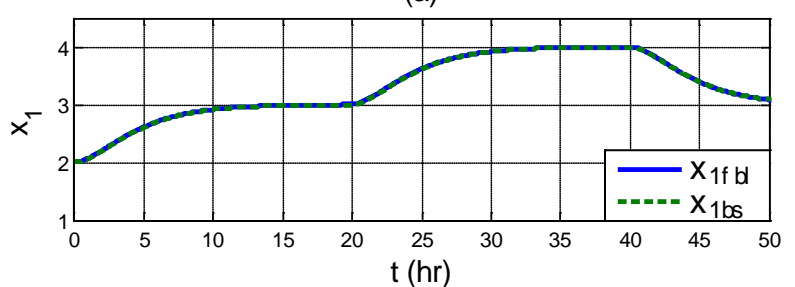

(b)

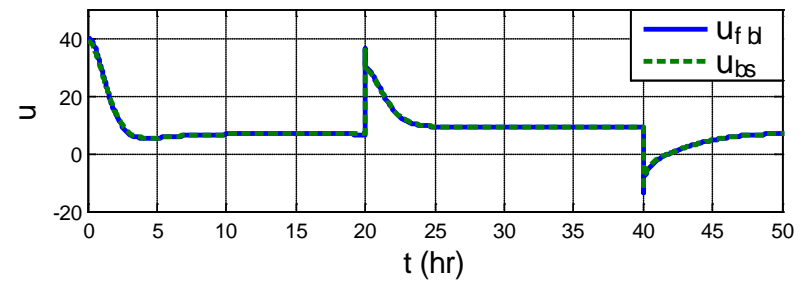

Figure 4. Zoomed-in view of $x_{1 f b l}(t)$ and $x_{1 b s}(t)$ and $u_{f b l}(t)$ and $u_{b s}(t)$ in Figure 3.

investigate the constructions of Luenberger observers for the FBL-based and BS-based control approaches in this section

\subsection{Luenberger Observer for FBL Control}

Since only $x_{1}(t)$ is measured in (4), a Luenberger observer [12] can be constructed for full-state estimation needed for full-state control of the bioprocess system. Using (21), a full-state observer can be constructed as

$$
\left\{\begin{array}{l}
\dot{\hat{\mathbf{z}}}=\left(\boldsymbol{A}_{c}-\boldsymbol{B}_{c} \boldsymbol{K}_{f b l}\right) \hat{\mathbf{z}}+\boldsymbol{B}_{c}\left(\boldsymbol{K}_{f b l} \boldsymbol{Y}_{r}+\ddot{y}_{r}\right)+\boldsymbol{L}(y-\hat{y})=\boldsymbol{A}_{o} \hat{\mathbf{z}}+\boldsymbol{B}_{c}\left(\boldsymbol{K}_{f b l} \boldsymbol{Y}_{r}+\ddot{y}_{r}\right)+\boldsymbol{L} y, \\
y=\boldsymbol{C}_{c} \mathbf{z}=z_{1}=x_{1}, \quad \hat{y}=\boldsymbol{C}_{c} \hat{\mathbf{z}}=\hat{z}_{1}=\hat{x}_{1},
\end{array}\right.
$$

where $\boldsymbol{A}_{o} \triangleq \boldsymbol{A}_{c}-\boldsymbol{B}_{c} \boldsymbol{K}_{f b l}-\boldsymbol{L C}_{c}$ is the observer system matrix and $\boldsymbol{L} \in \mathbb{R}^{2 \times 1}$ the observer gain matrix to be determined such that $\boldsymbol{A}_{o}$ is Hurwitz, provided that $\left[\left(\boldsymbol{A}_{c}-\boldsymbol{B}_{c} \boldsymbol{K}_{f b l}\right), \boldsymbol{C}_{c}\right]$ is an observable pair (which is the case in the present problem). The gain matrix $\boldsymbol{L}$ in (42) can be computed using a Luenberger observer [12] with pole placement (PP) and/or Kalman-Bucy filter [13] design techniques. We shall focus on the PP design method; 
henceforth $\boldsymbol{L}$ and $\boldsymbol{A}_{o}=\boldsymbol{A}_{c}-\boldsymbol{B}_{c} \boldsymbol{K}_{f b l}-\boldsymbol{L} \boldsymbol{C}_{c}$ in (42) will be denoted by $\boldsymbol{L}=\boldsymbol{L}_{p p}$ and $\boldsymbol{A}_{o p p}=\boldsymbol{A}_{c}-\boldsymbol{B}_{c} \boldsymbol{K}_{f b l}-\boldsymbol{L}_{p p} \boldsymbol{C}_{c}$, respectively. It should be noted that for a general LTI system characterized by $[\boldsymbol{A}, \boldsymbol{B}, \boldsymbol{C}]$, where $[\boldsymbol{A}, \boldsymbol{C}]$ is an observable pair, the pair $[(\boldsymbol{A}-\boldsymbol{B K}), \boldsymbol{C}]$ may not be observable, because full-state feedback can destroy observability; furthermore, $(\boldsymbol{A}-\boldsymbol{B K}-\boldsymbol{L C})$ may be unstable even though $(\boldsymbol{A}-\boldsymbol{L C})$ is designed to be stable [14] [15].

Now using (21) and (42), it can readily be shown that the estimation error $\tilde{\boldsymbol{z}}=\mathbf{z}-\hat{\mathbf{z}}$ satisfies,

$$
\dot{\tilde{\mathbf{z}}}=\boldsymbol{A}_{\text {opp }} \tilde{\mathbf{z}}, \quad \tilde{\mathbf{z}}(0)=\tilde{\mathbf{z}}_{o},
$$

where the initial condition $\tilde{\boldsymbol{z}}(0)$ is arbitrary. Since $\boldsymbol{A}_{\text {opp }}$ is Hurwitz, it follows that

$$
\lim _{t \rightarrow \infty}[\tilde{\mathbf{z}}(t)]=\lim _{t \rightarrow \infty}[\mathbf{z}(t)-\hat{\mathbf{z}}(t)]=0
$$

for all $\tilde{\boldsymbol{z}}(0) \neq 0$.

Using the transformation defined by (17), the observer described by (42) in the $z$-coordinates can be transformed back to the $x$-coordinates as

$$
\dot{\hat{\boldsymbol{x}}}=\boldsymbol{Q}_{f b l}^{-1}(\hat{\boldsymbol{x}}) \dot{\hat{\mathbf{z}}}=\boldsymbol{f}(\hat{\boldsymbol{x}})+\boldsymbol{g}(\hat{\boldsymbol{x}}) \hat{u}_{f b l}+\boldsymbol{Q}_{f b l}^{-1}(\hat{\boldsymbol{x}}) \boldsymbol{L}_{p p}\left(y-\hat{x}_{1}\right),
$$

where $\boldsymbol{Q}_{f b l}^{-1}(\hat{\boldsymbol{x}}) \triangleq\left(\frac{\partial \boldsymbol{T}_{f b l}}{\partial \boldsymbol{x}}(\hat{\boldsymbol{x}})\right)^{-1}$ and $\boldsymbol{Q}_{f b l}(\hat{\boldsymbol{x}})$ is the Jacobian matrix associated with (17) given by

$$
\boldsymbol{Q}_{f b l}(\hat{\boldsymbol{x}})=\left[\begin{array}{cc}
1 & 0 \\
\frac{C_{1} \hat{x}_{2}}{K_{s}+\hat{x}_{2}}-1 & \frac{C_{1} K_{s} \hat{x}_{1}}{\left(K_{s}+\hat{x}_{2}\right)^{2}}
\end{array}\right] .
$$

In summary, the observer-based control system with feedback linearization for the bioprocess under consideration has the form

$$
\begin{gathered}
\dot{\boldsymbol{x}}=\boldsymbol{f}(\boldsymbol{x})+\boldsymbol{g}(\boldsymbol{x}) \hat{u}_{f b l}, \quad \boldsymbol{x}(0)=\boldsymbol{x}_{o}, \\
\dot{\hat{\boldsymbol{x}}}=\boldsymbol{f}(\hat{\boldsymbol{x}})+\boldsymbol{g}(\hat{\boldsymbol{x}}) \hat{u}_{f b l}+\boldsymbol{Q}_{f b l}^{-1}(\hat{\boldsymbol{x}}) \boldsymbol{L}_{p p}\left(y-\hat{x}_{1}\right), \quad \hat{\boldsymbol{x}}(0)=\hat{\boldsymbol{x}}_{o},
\end{gathered}
$$

where $\hat{\boldsymbol{x}}(0)$ is the initial estimate of $\boldsymbol{x}(0)$ and

$$
\begin{gathered}
\hat{u}_{f b l}=\beta^{-1}(\hat{\boldsymbol{x}})[-\alpha(\hat{\boldsymbol{x}})+\hat{v}], \\
\hat{v}=-\boldsymbol{K}_{f b l} \boldsymbol{T}_{f b l}(\hat{\boldsymbol{x}})+\boldsymbol{K}_{f b l} \boldsymbol{Y}_{r}+\ddot{y}_{r}, \\
\alpha(\hat{\boldsymbol{x}})=\frac{\left(C_{1} \hat{x}_{2}-K_{s}-\hat{x}_{2}\right)\left(C_{1} \hat{x}_{1} \hat{x}_{2}-K_{s} \hat{x}_{1}-\hat{x}_{1} \hat{x}_{2}\right)}{\left(K_{s}+\hat{x}_{2}\right)^{2}}+\frac{C_{1} C_{2} K_{s} \hat{x}_{1}^{2} \hat{x}_{2}}{\left(K_{s}+\hat{x}_{2}\right)^{3}}-\frac{C_{1} K_{s} \hat{x}_{1} \hat{x}_{2}}{\left(K_{s}+\hat{x}_{2}\right)^{2}}, \\
\beta(\boldsymbol{x})=\frac{C_{1} K_{S} \hat{x}_{1}}{\left(K_{s}+\hat{x}_{2}\right)^{2}} .
\end{gathered}
$$

\subsection{Luenberger Observer for BS Control}

In this section we pursue our final objective, i.e., to design a Luenberger observer based on the BS formulation using the error Equation (40). To construct an observer for (40), we need an output equation which can be defined as,

$$
\tilde{y}=q_{1}=\left[\begin{array}{ll}
1 & 0
\end{array}\right] \boldsymbol{q} \triangleq \boldsymbol{C}_{q} \boldsymbol{q},
$$

where $\tilde{y}=q_{1}=x_{1}-y_{r}$ is known and $\left[\boldsymbol{A}_{q}, \boldsymbol{C}_{q}\right]$ is an observable pair. 
We present the following proposition.

\section{Proposition 1}

Consider the bioprocess system described by (3) and (4). A Luenberger observer for the associated error system (40) with measurement given by (53) can be constructed as

$$
\dot{\hat{\boldsymbol{q}}}=\boldsymbol{A}_{q} \hat{\boldsymbol{q}}+\boldsymbol{L}_{b s}\left(\tilde{y}-\boldsymbol{C}_{q} \hat{\boldsymbol{q}}\right), \quad \hat{\boldsymbol{q}}(0)=\hat{\boldsymbol{q}}_{o},
$$

where $\tilde{y}-\boldsymbol{C}_{q} \hat{\boldsymbol{q}}=y-\hat{x}_{1}$ and $\boldsymbol{L}_{b s} \in \mathbb{R}^{2 \times 1}$ is the observer gain matrix to be determined by, for example poleplacement, such that the observer matrix $\boldsymbol{A}_{o b s}=\boldsymbol{A}_{q}-\boldsymbol{L}_{b s} \boldsymbol{C}$ is Hurwitz. Since $\boldsymbol{A}_{q}$ is already Hurwitz and $\left[\boldsymbol{A}_{q}, \boldsymbol{C}_{q}\right]$ is an observable pair, $\boldsymbol{L}_{b s}$ can be determined such that the real parts of the eigenvalues of $\boldsymbol{A}_{o b s}=\boldsymbol{A}_{q}-\boldsymbol{L}_{b s} \boldsymbol{C}_{q}$ lie on the left-side of those of $\boldsymbol{A}_{q}$ on the open left-half plane, if desirable. Furthermore, (54) can be expressed in the $x$-coordinates as

$$
\dot{\hat{\boldsymbol{x}}}=\boldsymbol{f}(\hat{\boldsymbol{x}})+\boldsymbol{g}(\hat{\boldsymbol{x}}) \hat{u}_{b s}+\boldsymbol{Q}_{b s}^{-1} \boldsymbol{L}_{b s}\left(y-\hat{x}_{1}\right), \quad \hat{\boldsymbol{x}}(0)=\hat{\boldsymbol{x}}_{o} .
$$

Proof: First, we need to show that the estimate $\hat{\boldsymbol{q}}$ converges to its true value $\boldsymbol{q}$. Define the estimation error as $\tilde{\boldsymbol{q}}=\boldsymbol{q}-\hat{\boldsymbol{q}}$. From (40) and (54), it follows that $\tilde{\boldsymbol{q}}$ satisfies,

$$
\dot{\tilde{\boldsymbol{q}}}=\dot{\boldsymbol{q}}-\dot{\hat{\boldsymbol{q}}}=\left(\boldsymbol{A}_{q}-\boldsymbol{L}_{b s} \boldsymbol{C}_{q}\right)(\boldsymbol{q}-\hat{\boldsymbol{q}}) \triangleq \boldsymbol{A}_{o b s} \tilde{\boldsymbol{q}}, \quad \tilde{\boldsymbol{q}}(0)=\tilde{\boldsymbol{q}}_{o},
$$

where $\tilde{\boldsymbol{q}}(0)$ is the initial condition. Since $\boldsymbol{A}_{\text {obs }}$ is Hurwitz, it follows that

$$
\lim _{t \rightarrow \infty}[\tilde{\boldsymbol{q}}]=\lim _{t \rightarrow \infty}[\boldsymbol{q}-\hat{\boldsymbol{q}}]=0,
$$

for arbitrary $\tilde{\boldsymbol{q}}(0)$ Next, using, (24), (25) and (29), the coordinates transformation for the error-system can be obtained as

$$
\boldsymbol{q}=\left[\begin{array}{c}
x_{1}-y_{r} \\
\theta_{1}^{-1} r\left(x_{2}\right) x_{1}-\alpha_{1}
\end{array}\right] \triangleq \boldsymbol{T}_{b s}\left(\boldsymbol{x}, y_{r}, \dot{y}_{r}\right)=\left[\begin{array}{c}
x_{1}-y_{r} \\
\theta_{1}^{-1} r\left(x_{2}\right) x_{1}+\left(c_{1}-1\right) x_{1}-c_{1} y_{r}-\dot{y}_{r}
\end{array}\right] .
$$

Equation (58) yields the Jacobian matrix

$$
\boldsymbol{Q}_{b s}(\boldsymbol{x}) \triangleq \frac{\partial \boldsymbol{T}_{b s}}{\partial \boldsymbol{x}}=\left[\begin{array}{cc}
1 & 0 \\
\theta_{1}^{-1} r\left(x_{2}\right)+\left(c_{1}-1\right) & \theta_{1}^{-1} \frac{\partial r}{\partial x_{2}} x_{1}
\end{array}\right],
$$

where $\boldsymbol{Q}_{b s}$ is nonsingular for $\theta_{1}^{-1} \frac{\partial r}{\partial x_{2}} x_{1} \neq 0$ so that $\boldsymbol{T}_{b s}\left(\boldsymbol{x}, y_{r}, \dot{y}_{r}\right)$ is a local diffeomorphism for (3). Equations (58) and (59) yield $\dot{\hat{\boldsymbol{q}}}=\boldsymbol{Q}_{b s}(\hat{\boldsymbol{x}}) \dot{\hat{\boldsymbol{x}}}$ and, from (53) and (54),

$$
\dot{\hat{\boldsymbol{x}}}=\boldsymbol{Q}_{b s}^{-1}(\hat{\boldsymbol{x}}) \dot{\hat{\boldsymbol{q}}}=\boldsymbol{Q}_{b s}^{-1}(\hat{\boldsymbol{x}})\left[\boldsymbol{A}_{q} \hat{\boldsymbol{q}}+\boldsymbol{L}_{b s}\left(y-\hat{x}_{1}\right)\right]=\boldsymbol{f}(\hat{\boldsymbol{x}})+\boldsymbol{g}(\hat{\boldsymbol{x}}) \hat{u}_{b s}+\boldsymbol{Q}_{b s}^{-1}(\hat{\boldsymbol{x}}) \boldsymbol{L}_{b s}\left(y-\hat{\boldsymbol{x}}_{1}\right),
$$

where $\boldsymbol{Q}_{b s}^{-1}(\hat{\boldsymbol{x}})$ is the inverse of $\boldsymbol{Q}_{b s}(\hat{\boldsymbol{x}})$, and

$$
\begin{gathered}
\hat{u}_{b s}=\left(\theta_{1}^{-1} \hat{x}_{1} \frac{\partial r}{\partial \hat{x}_{2}}\right)^{-1}\left[-c_{2} \hat{q}_{2}-\hat{q}_{1}-\phi(\hat{x})+\dot{\hat{\alpha}}_{1}\right], \\
\dot{\hat{\alpha}}_{1}=-c_{1} \dot{\hat{q}}_{1}+\dot{\hat{x}}_{1}+\ddot{y}_{r}=-c_{1}\left(-c_{1} \hat{q}_{1}+\hat{q}_{2}\right)+\theta_{1}^{-1} r\left(\hat{x}_{2}\right) \hat{x}_{1}-\hat{x}_{1}+\ddot{y}_{r}, \quad c_{1}>0,
\end{gathered}
$$

which complete the proof.

The observer design technique developed here is interesting and attractive and is different from the two-filter approach in [9]. The technique can be applied to a wide class of BS-based error systems.

In summary, the observer-based control system with the BS formulation for the bioprocess is described by

$$
\dot{\boldsymbol{x}}=\boldsymbol{f}(\boldsymbol{x})+\boldsymbol{g}(\boldsymbol{x}) \hat{u}_{b s}, \quad \boldsymbol{x}(0)=\boldsymbol{x}_{o},
$$




$$
\dot{\hat{\boldsymbol{x}}}=\boldsymbol{f}(\hat{\boldsymbol{x}})+\boldsymbol{g}(\hat{\boldsymbol{x}}) \hat{u}_{b s}+\boldsymbol{Q}_{b s}^{-1}(\hat{\boldsymbol{x}}) \boldsymbol{L}_{b s}\left(y-\hat{x}_{1}\right), \quad \hat{\boldsymbol{x}}(0)=\hat{\boldsymbol{x}}_{o},
$$

where $\hat{\boldsymbol{x}}(0)$ is the initial estimate of $\boldsymbol{x}(0)$.

Simulation studies for the proposed observer-based FBL and BS controls were conducted and compared. The initial conditions were chosen as $\boldsymbol{x}(0)=\left[\begin{array}{ll}2 & 0.9\end{array}\right]^{\mathrm{T}}$ and $\hat{\boldsymbol{x}}(0)=\left[\begin{array}{ll}0.8 & 0.1\end{array}\right]^{\mathrm{T}}$. The set-point reference $y_{r}(t)$ was the same as before. The model parameters were given in Table 1 and Table 2.

In Figure 5, results for observer-based FBL control scheme described by (47) and (48) are shown. It can be seen that the estimates $\hat{x}_{1 f b l}(t) \rightarrow x_{1}(t)$ and $\hat{x}_{2 f b l}(t) \rightarrow x_{2}(t)$ converged to the true states around $t=7 \mathrm{~h}$. In Figure 6, results for the observer-based BS control scheme are presented. Convergence of the estimated states to the actual states can also be seen from this figure, and are similar to those presented in Figure 2.

In Figure 7, the behavior of the error variables $q_{1}$ and $q_{2}$ defined by (24) and (25) which satisfy (40) in the backstepping scheme is shown. It is evident that $\hat{q}_{1}(t) \rightarrow q_{1}(t)$ and $\hat{q}_{2}(t) \rightarrow q_{2}(t)$ smoothly after the transients are over around $t=8 \mathrm{~h}$.

\section{Conclusion}

Observers are critical to control system analysis and designs that employ full-state feedback, where not all the state variables are accessible for on-line, real-time measurements, and/or where the measurements are corrupted by noise. Indeed, the design of suitable linear or nonlinear observers or filters leading to observer-based control technology is an integral part of real world control system applications. In this paper, observer-based control strategies were developed for a nonlinear bioprocess system using feedback linearization and backstepping control techniques; in particular, a Luenberger observer for backstepping control was formulated using the error equation resulted from the backstepping design procedure. The observer design technique developed here is interesting and attractive and is different from the two-filter approach known in the literature. Simulation results with and without observers for both the FBL and BS schemes are presented and compared. The results were excellent and demonstrated the feasibility and effectiveness of the proposed approaches.

Table 2. Controllers and observer gains.

\begin{tabular}{ccc}
\hline Observer-Based Control & Controller Gain/Coefficients & Observer Gain $\boldsymbol{L}$ \\
\hline FBL & $\boldsymbol{K}_{b l}=\left[\begin{array}{ll}20 & 9\end{array}\right]$ & $\boldsymbol{L}_{b b l}=\left[\begin{array}{ll}19.4 & 92.13\end{array}\right]^{\mathrm{T}}$ \\
BS & {$\left[\begin{array}{ll}c_{1} & c_{2}\end{array}\right]=\left[\begin{array}{ll}3.5 & 3.5\end{array}\right]$} & $\boldsymbol{L}_{b s}=\left[\begin{array}{ll}12.4 & 35.48\end{array}\right]^{\mathrm{T}}$ \\
\hline
\end{tabular}

(a)
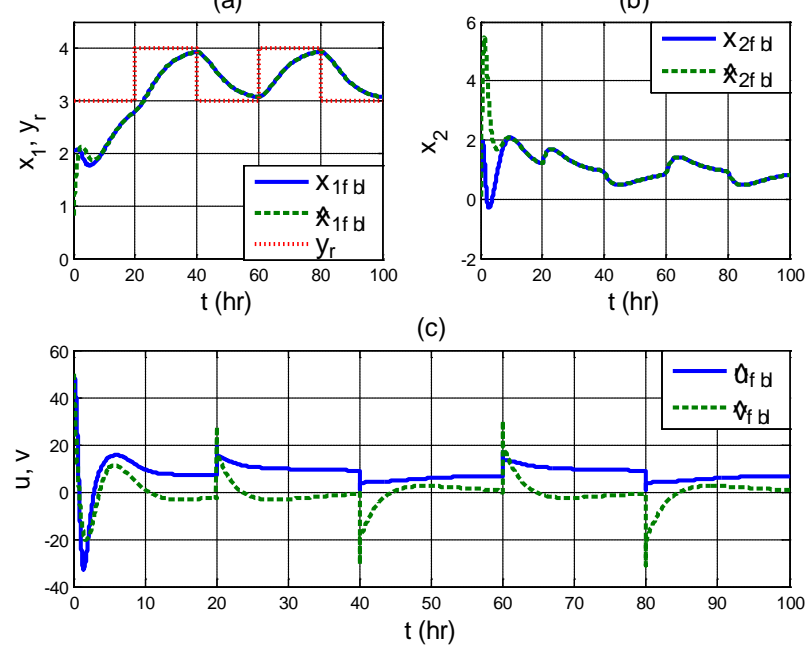

Figure 5. Responses of observer-based FBL control scheme (47) and (48): $\hat{x}_{1 f b l}(t) \rightarrow x_{1}(t)$ and $\hat{x}_{2 f b l}(t) \rightarrow x_{2}(t)$ smoothly.

(b)

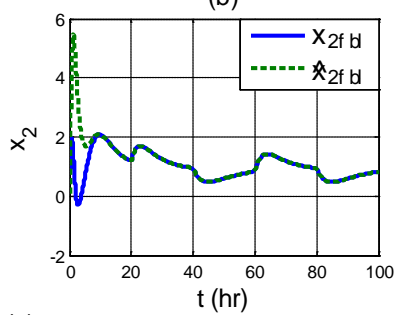

(c)

\section{(1)}


(a)

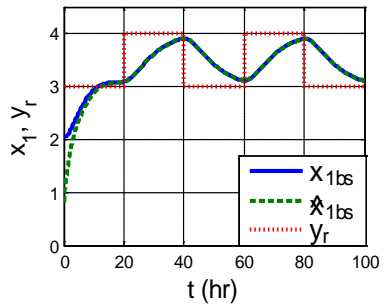

(b)

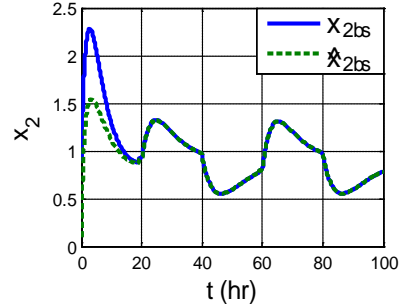

(c)

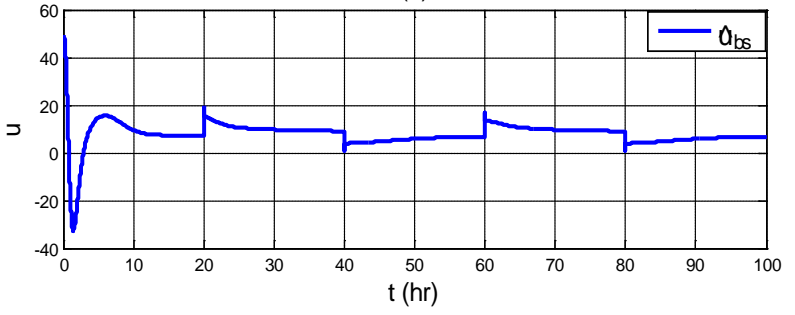

Figure 6. Responses of observer-based BS control scheme (63) and (64): $\hat{x}_{1 b s}(t) \rightarrow x_{1}(t)$ and $\hat{x}_{2 b s}(t) \rightarrow x_{2}(t)$ smoothly.

(a)

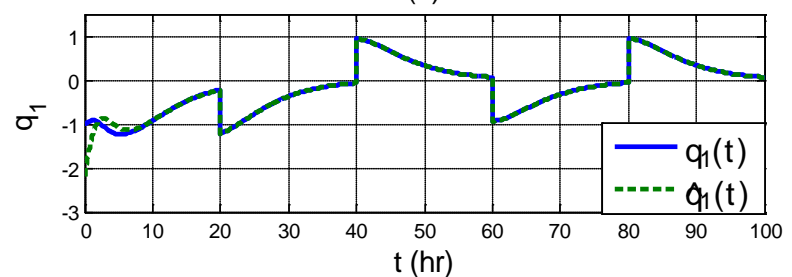

(b)

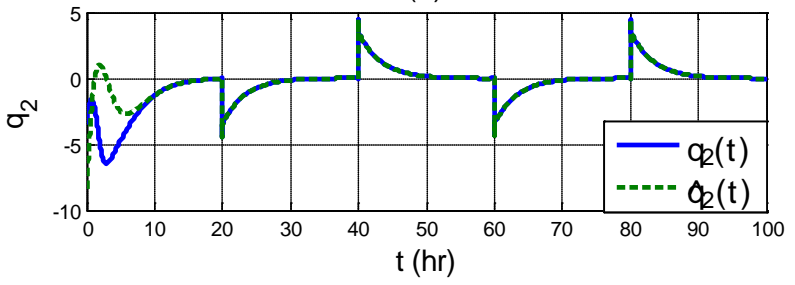

Figure 7. Evolution of the backstepping error variables: $\hat{q}_{1}(t) \rightarrow q_{1}(t)$ and $\hat{q}_{2}(t) \rightarrow q_{2}(t)$ smoothly.

\section{Acknowledgements}

The authors would like to thank all the reviewers for their feedbacks and constructive criticisms.

\section{References}

[1] Bastin, G. and Dochain, D. (1990) On-line Estimation and Adaptive Control of Bioreactors. Elsevier, Amsterdam.

[2] Dochain, D. (2003) State and Parameter Estimation in Chemical and Biochemical Processes: A Tutorial. Journal of Process Control, 13, 801-818. http://dx.doi.org/10.1016/S0959-1524(03)00026-X

[3] Dochain, D. and Rapaport, A. (2005) Internal Observers for Biochemical Processes with Uncertain Kinetics and Inputs. Mathematical Biosciences, 193, 235-253. http://dx.doi.org/10.1016/j.mbs.2004.07.004

[4] Dochain, D. (2000) State Observers for Tubular Reactors with Unknown Kinetics. Journal of Process Control, 10, 259-268. http://dx.doi.org/10.1016/S0959-1524(99)00020-7

[5] Hulhoven, X., Vande Wouwer, A. and Bogaerts, Ph. (2006) Hybrid Extended Luenberger-Asymptotic Observer for Bioprocess State Estimation. Chemical Engineering Science, 61, 7151-7160.

http://dx.doi.org/10.1016/j.ces.2006.06.018 
[6] Dochain, D. and Perrier, M. (2003) Adaptive Backstepping Nonlinear Control of Bioprocesses. International Federation of Automatic Control Proceedings, ADCHEM, 77-82.

[7] Khalil, H.K. (2002) Nonlinear Systems. 3rd Edition, Prentice Hall, Upper Saddle River.

[8] Marquez, H.J. (2003) Nonlinear Control Systems: Analysis and Design. John Wiley \& Sons, Hoboken.

[9] Krstic, M., Kanellakopoulos, I. and Kokotovic, P. (1995) Nonlinear and Adaptive Control Design. John Wiley, New York.

[10] Isidori, A. (1995) Nonlinear Control Systems. Springer-Verlag, New York. http://dx.doi.org/10.1007/978-1-84628-615-5

[11] Krener, A.J. (1999) Feedback Linearization. In: Baillieul, J. and Willems, J.C., Eds. Mathematical Control Theory, Chapter 3, Springer-Verlag, New York, 66-98.

[12] Luenberger, D.G. (1964) Observing the State of a Linear System. IEEE Transactions on Military Electronics, 8, 74-80. http://dx.doi.org/10.1109/TME.1964.4323124

[13] Kalman, R.E. and Bucy, R.S. (1961) New Results in Linear Filtering and Prediction Theory. Transactions of the ASME, 83D, 429-438.

[14] Ogata, K. (2010) Modern Control Systems. 5th Edition, Prentice Hall, Upper Saddle River.

[15] Dorf, R. and Bishop, R. (2008) Modern Control Systems. 11th Edition, Pearson Education, Upper Saddle River. 
Scientific Research Publishing (SCIRP) is one of the largest Open Access journal publishers. It is currently publishing more than 200 open access, online, peer-reviewed journals covering a wide range of academic disciplines. SCIRP serves the worldwide academic communities and contributes to the progress and application of science with its publication.

Other selected journals from SCIRP are listed as below. Submit your manuscript to us via either submit@scirp.org or Online Submission Portal.
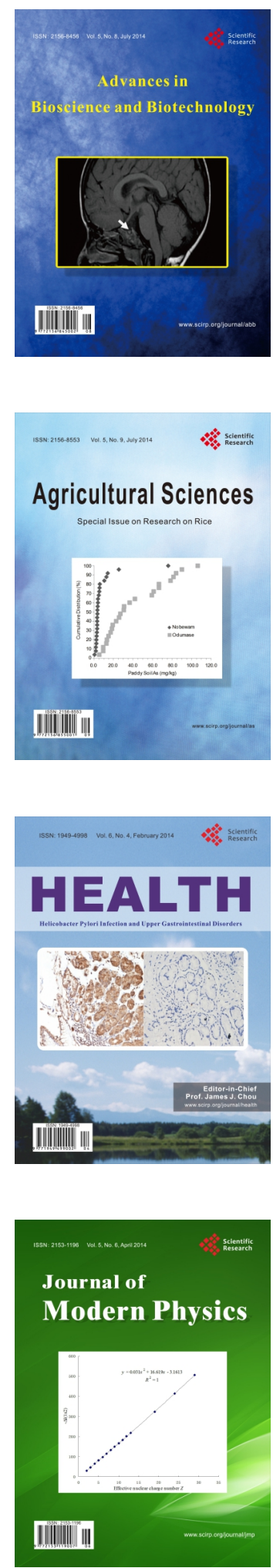
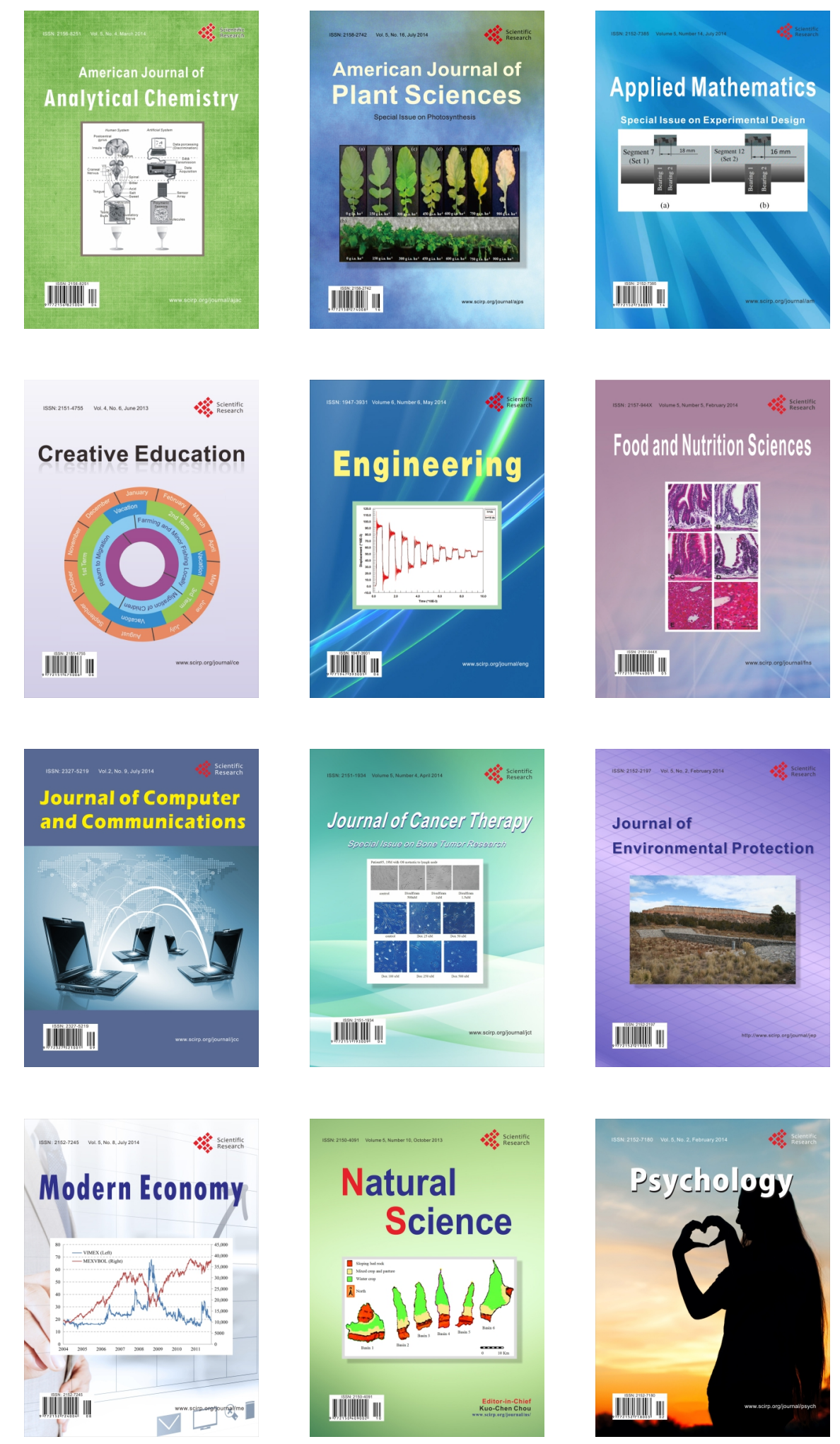